Check for updates

Cite this: RSC Adv., 2019, 9, 11755

\title{
Hydrogen evolution reaction activity related to the facet-dependent electrocatalytic performance of NiCoP from first principles $\uparrow$
}

\begin{abstract}
Jie Mou, ${ }^{\text {ab }}$ Yuyue Gao, ${ }^{\text {ab }}$ Jingbo Wang, ${ }^{b}$ Jianyi Ma (iD ${ }^{a}$ and Haisheng Ren (D)*b
Transition metal phosphides (TMPs) have been proven to act as highly active catalysts for the hydrogen evolution reaction (HER). Recently, single-phase ternary NiCoP electrocatalysts have been shown through experiments to display remarkable catalytic activity for the HER during water splitting. But, the inherent mechanism is not well understood. Herein, the HER activity of NiCoP with low-Miller-index facets, including (111), (100), (001)-NiP-t, and (001)-CoP-t, was systematically investigated using periodic density functional theory (DFT). The calculated Gibbs free energy of hydrogen adsorption $\left(\Delta G_{H}\right)$ values reveal that all calculated facets have good catalytic activity for the HER. The (111) facet with the lowest surface energy in a vacuum has optimal $\Delta G_{H}$ values close-to-zero for a range of hydrogen coverage. $A b$ initio thermodynamic analysis of hydrogen coverage was conducted to obtain the stabilities of surfaces, which follow the trend: (111) > (001)-CoP-t > (100) > (001)-NiP-t at $1 \mathrm{~atm} \mathrm{H}_{2}$ and $298 \mathrm{~K}$. We hope that this work can shed new light on further understanding the HER in relation to NiCoP and can give guidance for the design and synthesis of transition bimetal phosphide-based catalysts.
\end{abstract}

Received 1st March 2019

Accepted 1st April 2019

DOI: 10.1039/c9ra01560d

rsc.li/rsc-advances the years, some promising HER materials have been developed, such as sulfides, ${ }^{7,8}$ borides, ${ }^{9}$ selenides, ${ }^{10}$ carbides, ${ }^{11,12}$ and nitrides, ${ }^{13}$ as substitutes for benchmark platinum materials. However, these satisfactory achievements did not meet the needs of industrial progress. Transition metal phosphides (TMPs), as a consequence, have received much interest from researchers worldwide due to their high catalytic activities for the HER compared to other non-precious electrocatalysts. ${ }^{\mathbf{1 4 - 1 6}}$ For example, TMPs, including phosphides of molybdenum, ${ }^{17,18}$ iron, $^{19,20}$ cobalt, $^{21,22}$ and nickel, ${ }^{23,24}$ are well known as hydrogenase-like catalysts, with high catalytic activity and durability. In particular, ternary $\mathrm{Ni}_{2-x} \mathrm{Co}_{x} \mathrm{P}$ systems, which have been researched extensively, have been shown to be highly efficient catalysts for the HER and oxygen evolution reaction (OER). ${ }^{25-31}$ Among these systems, single-phase ternary NiCoP nanocrystals deliver near-optimal HER catalytic activity, with an overpotential as low as $59 \mathrm{mV}$ and reaching a catalytic current density of $10 \mathrm{~mA} \mathrm{~cm}^{-2}$. In addition, the derived Tafel slope of the NiCoP nanocrystals is about $50 \mathrm{mV} \mathrm{dec}^{-1}$, approaching that of $\mathrm{Pt} / \mathrm{C}\left(30 \mathrm{mV} \mathrm{dec}{ }^{-1}\right) .{ }^{32}$ Although there are so many exciting experimental results relating to $\mathrm{NiCoP}$, insights into ternary NiCoP for systematic hydrogen absorption are lacking, which is of critical importance for further enhancing the activities and stabilities of HER electrocatalysts.

The Volmer reaction involves a proton from an acidic solution bonding to an active site on a facet to form $\mathrm{H}^{\cdot}\left(\mathrm{H}^{+}+\mathrm{e}^{-}+\right.$ $\left.\rightarrow \mathrm{H}^{\cdot}\right)^{33}$ (here, ${ }^{\cdot}$ represents an active site on the facet and $\mathrm{H}^{\cdot}$ means adsorbed $\mathrm{H}$ ). Volmer-Heyrovsky and Volmer-Tafel processes are two types of possible pathways that have been 
proposed for the HER mechanism in acidic media. In the former, a solvated proton from the water layer reacts with $\mathrm{H}^{*}$ to form $\mathrm{H}_{2}\left(\mathrm{H}^{+}+\mathrm{e}^{-}+\mathrm{H}^{\cdot} \rightarrow \mathrm{H}_{2}+^{\cdot}\right),{ }^{34}$ while in the latter, two adsorbed hydrogen atoms react with each other to form a $\mathrm{H}_{2}$ molecule $\left(\mathrm{H}^{\cdot}+\mathrm{H}^{\cdot} \rightarrow \mathrm{H}_{2}+2^{\circ}\right) .^{35}$ Therefore, good electrocatalysts for the HER should have the advantages of both being able to attract protons from solution and desorb $\mathrm{H}_{2}$ molecules.

The Gibbs free energy of hydrogen adsorption $\left(\Delta G_{\mathrm{H}}\right)$ is regarded as the most important factor for describing the HER activity of an electrocatalyst. Parsons ${ }^{36}$ first proposed that the optimal HER activity of an electrocatalyst should have a $\Delta G_{\mathrm{H}}$ value of around zero. Subsequently, Nørskov et al. ${ }^{37,38}$ and other groups ${ }^{7,8,16}$ combined experiment and theory to further prove this. When the value of $\Delta G_{\mathrm{H}}$ equals zero, the maximum HER activity is obtainable. A more negative value of $\Delta G_{\mathrm{H}}$ results in stronger binding between hydrogen and the facets, which will impede the desorption of $\mathrm{H}_{2}$ molecules. On the other hand, a more positive value of $\Delta G_{\mathrm{H}}$ will result in weaker binding between hydrogen and the facets, which will hinder the proton/ electron-transfer step. Thus, efficient HER electrocatalysts can be obtained at a $\Delta G_{\mathrm{H}}$ value close to zero.

In order to figure out the HER mechanisms on NiCoP, the absorption structures and energetics of atomic hydrogen on four low-Miller-index facets of NiCoP have been systematically investigated using periodic DFT in this work. We use the calculated $\Delta G_{\mathrm{H}}$ values to predict the HER activities of the surfaces. In addition, $a b$ initio atomistic thermodynamics was employed to determine the most stable and active facet of $\mathrm{NiCoP}$ at $1 \mathrm{~atm} \mathrm{H}_{2}$ pressure and $298 \mathrm{~K}$. It is expected that our results can provide useful insights and guidelines for designing transition bimetal phosphide HER electrocatalysts.

\section{Computational methods}

Spin-polarized periodic DFT calculations were performed via the plane-wave technique implemented in the Vienna ab initio simulation package (VASP). ${ }^{39}$ The ion-electron interactions were described with the projector augmented plan wave (PAW) method, ${ }^{\mathbf{4 0 , 4 1}}$ and the exchange-correlation energies of interacting electrons were represented using the Perdew-Burke-Ernzerhof (PBE) functional with generalized gradient approximation (GGA). ${ }^{42}$ The Gaussian smearing method was adopted to describe the total energy, with a smearing width of $0.02 \mathrm{eV}$. A plane-wave cutoff energy was tested and set to $400 \mathrm{eV}$. Structure optimization was continued until the residual force converged to less than $0.01 \mathrm{eV}^{-1}$ and the total energy converged to less than $1.0 \times 10^{-6} \mathrm{eV}$. Slabs more than $10 \AA$ thick in $(2 \times 2)$ supercells, with $15 \AA$ of vacuum along the perpendicular catalyst facets, were used to build the adsorbate-facet systems for (111), (100), (001)-NiP-t and (001)-CoP-t facets. A gamma $k$-point of $7 \times 7 \times 12$ and a Monkhorst-Pack $k$-point mesh of $3 \times 3 \times 1$ were set to optimize bulk NiCoP and the four adsorbate-facet systems of NiCoP, respectively. The added hydrogen atoms together with the top two layers were allowed to move during the geometry optimization over the whole calculation.

The surface energy was defined as

$$
\gamma=\frac{E_{\text {slab }}-N E_{\text {bulk }}}{2 A}
$$

where $E_{\text {slab }}$ is the total energy of the facet slab, $E_{\text {bulk }}$ is the total energy of a bulk unit cell of NiCoP, $N$ is the number of formula units in the slab and $A$ is the facet area of the optimized slab. The hydrogen adsorption energy $\Delta E_{\mathrm{H}}$ was calculated using

$$
\Delta E_{\mathrm{H}}=E(\mathrm{NiCoP}+n \mathrm{H})-E[\mathrm{NiCoP}+(n-1) \mathrm{H}]-\frac{1}{2} E\left(\mathrm{H}_{2}\right)
$$

where $E(\mathrm{NiCoP}+n \mathrm{H})$ and $E[\mathrm{NiCoP}+(n-1) \mathrm{H}]$ stand for the total energy of NiCoP with $n$ and $n-1$ hydrogen atoms adsorbed on the facet. $E\left(\mathrm{H}_{2}\right)$ is the total energy of a gas phase $\mathrm{H}_{2}$ molecule. The Gibbs free energy of adsorbed $\mathrm{H}$ was obtained using

$$
\Delta G_{\mathrm{H}}=\Delta E_{\mathrm{H}}+\Delta E_{\mathrm{ZPE}}-T \Delta S_{\mathrm{H}}
$$

where $\Delta E_{\mathrm{ZPE}}$ and $\Delta S_{\mathrm{H}}$ represent the zero-point energy change and entropy change between adsorbed hydrogen and hydrogen in the gas phase under standard conditions, respectively. $T$ is the temperature. $\Delta S_{\mathrm{H}}$ is approximately equal to $-\frac{1}{2} \Delta S_{\mathrm{H}_{2}}$, where $\Delta S_{\mathrm{H}_{2}}$ is the entropy of an isolated $\mathrm{H}_{2}$ molecule in the gas phase under standard conditions. Thus, the value of $T \Delta S_{\mathrm{H}}$ is close to -0.2 eV. $\Delta E_{\mathrm{ZPE}}$ can be expressed as

$$
\Delta E_{\mathrm{ZPE}}=E_{\mathrm{ZPE}}^{n \mathrm{H}}-E_{\mathrm{ZPE}}^{(n-1) \mathrm{H}}-\frac{1}{2} E_{\mathrm{ZPE}}^{\mathrm{H}_{2}}
$$

where $E_{\mathrm{ZPE}}^{n \mathrm{H}}, E_{\mathrm{ZPE}}^{(n-1) \mathrm{H}}$ and $E_{\mathrm{ZPE}}^{\mathrm{H}_{2}}$ are the zero-point energies of $n$ and ( $n-1)$ adsorbed hydrogen atoms, as well as a gas phase hydrogen molecule, respectively. The zero-point energy can be given as

$$
E_{\mathrm{ZPE}}=\sum_{1}^{i} \frac{1}{2} h \nu_{i}
$$

where $h$ is the Planck constant and $\nu_{i}$ is the vibrational frequency. For a single hydrogen molecule, the calculated value of the vibrational frequency is $4301 \mathrm{~cm}^{-1}$, which is in good agreement with the experimental finding of $4395 \mathrm{~cm}^{-1} \cdot{ }^{43}$ The calculated vibrational frequencies for hydrogen adsorbed on the four NiCoP facets are listed in Table S1. $\dagger$ According to eqn (4) and (5), the obtained values of $\Delta E_{\mathrm{ZPE}}$ are $0.04 \mathrm{eV}, 0.04 \mathrm{eV}$, $0.02 \mathrm{eV}$ and $0.03 \mathrm{eV}$ for the (111), (100), (001)-NiP-t and (001)CoP-t facets, respectively. Thus, the values of $\Delta G_{\mathrm{H}}$ can be rewritten as $\Delta G_{\mathrm{H}}=\Delta E_{\mathrm{H}}+0.24, \Delta G_{\mathrm{H}}=\Delta E_{\mathrm{H}}+0.24, \Delta G_{\mathrm{H}}=\Delta E_{\mathrm{H}}+$ 0.22 and $\Delta G_{\mathrm{H}}=\Delta E_{\mathrm{H}}+0.23$ for the (111), (100), (001)-NiP-t and (001)-CoP-t facets, respectively.

$A b$ initio atomistic thermodynamics ${ }^{44}$ was used to identify the most stable phase of each facet at 1 atm $\mathrm{H}_{2}$ and $298 \mathrm{~K} . \Delta G_{\mathrm{H}}$ at a specific temperature and pressure, $\Delta G^{\mathrm{ad}}(T, P)$, was calculated using

$$
\begin{aligned}
\Delta G^{\mathrm{ad}}(T, P)= & \frac{1}{A}\left[E^{\mathrm{total}}\left(N_{\mathrm{H}}\right)-E^{\mathrm{total}}(0)-\frac{N_{\mathrm{H}}}{2} E_{\mathrm{H}_{2}}^{\text {total }}\right. \\
& \left.-N_{\mathrm{H}} \Delta \mu_{\mathrm{H}}(T, P)\right]
\end{aligned}
$$

where $E^{\text {total }}\left(N_{\mathrm{H}}\right), E^{\text {total }}(0), E_{\mathrm{H}_{2}}^{\text {total }}$ and $\Delta \mu_{\mathrm{H}}(T, P)$ indicate the total energy of NiCoP with $N_{\mathrm{H}}$ hydrogen atoms adsorbed on a facet, 
the total energy of a clean facet, the energy of a gas phase $\mathrm{H}_{2}$ molecule, and the chemical potential of hydrogen at different temperatures and pressures, respectively. Obviously, the Gibbs free energy is a function of the hydrogen chemical potential for a particular surface at a particular $\mathrm{H}$ coverage. Therefore, the stability of $\mathrm{H}$-covered facets can be calculated at a given hydrogen chemical potential. $\Delta \mu_{\mathrm{H}}(T, P)$ is related to specific conditions using

$$
\Delta \mu_{\mathrm{H}}(T, P)=\Delta \mu_{\mathrm{H}}\left(T, P^{0}\right)+\frac{1}{2} k_{\mathrm{B}} T \ln \left(\frac{P}{P^{0}}\right)
$$

where $\Delta \mu_{\mathrm{H}}\left(T, P^{0}\right)$ is the hydrogen chemical potential at a standard pressure $P^{0}$ and $k_{\mathrm{B}}$ is the Boltzmann constant.

\section{Results and discussion}

\section{Surface energy and electronic density of states (DOS) for different facets}

The bulk NiCoP shown in Fig. 1 has a hexagonal $\mathrm{Fe}_{2} \mathrm{P}$-type structure with the symmetry group $P \overline{6} 2 \mathrm{~m}$. Each unit cell has three nickel atoms, three cobalt atoms and three phosphorous atoms. The equilibrium lattice parameters, together with available experimental data ${ }^{45}$ and recent calculations, ${ }^{32}$ from DFT with PBE functionals are listed in Table 1. The agreement is very good. The differences between the calculated and experimental findings do not exceed $0.03 \AA$.

Usually low Miller-index facets are considered preferentially in surface science studies due to their high durability. For hexagonal $\mathrm{Fe}_{2}$ P-type NiCoP, the low Miller-index facets can be cleaved to (111), (100) and (001) examples. For the (001) facet, with respect to hollow sites with three $\mathrm{Ni}$ or three Co atoms, there are two different truncation surfaces called (001)-NiP-t and (001)-CoP-t. The optimized structures of these facets are shown in Fig. 2 and the corresponding surface energies are listed in Table 2.

In general, more stable facets have lower surface energies and can be synthesized in experiments. ${ }^{46}$ Thus, the stability of the clean facets in a vacuum follow the order: $(111)>(001)-N i P-t$ $>$ (001)-CoP-t $>$ (100), based on computing the surface energy. The most stable example is the (111) facet, which agrees with
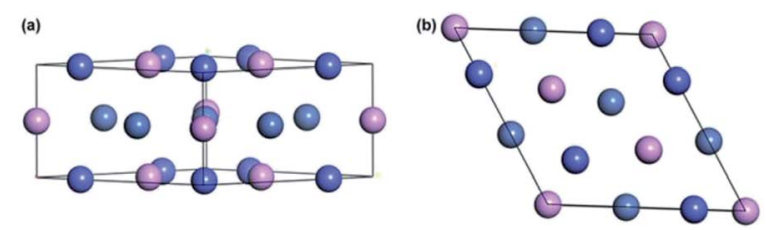

Fig. 1 Bulk NiCoP: (a) side view and (b) top view. Note that the colors gray, blue and pink stand for Ni, Co and $\mathrm{P}$ atoms, respectively.

Table 1 The lattice constants of bulk NiCoP

\begin{tabular}{llr}
\hline Bulk NiCoP & $a=b(\AA)$ & $c(\AA)$ \\
\hline Present & 5.80 & 3.35 \\
Calculated $^{32}$ & 5.82 & 3.36 \\
Experimental $^{45}$ & 5.83 & 3.35
\end{tabular}

(a)

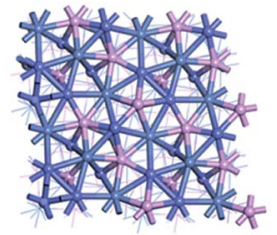

(c)

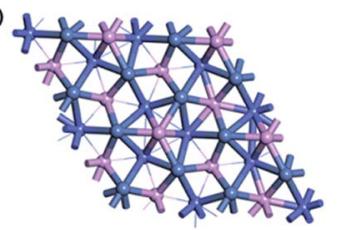

(b)
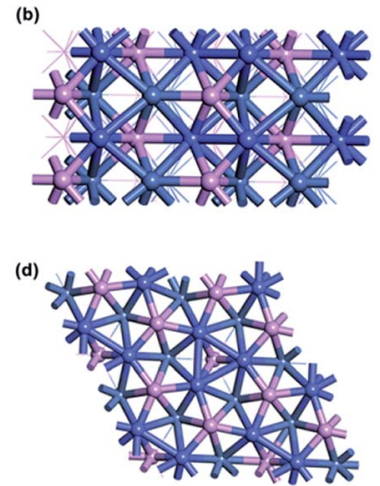

Fig. 2 Top views of clean NiCoP facets: (a) (111); (b) (100); (c) (001)NiP-t; and (d) (001)-CoP-t.

Table 2 Surface energies of clean NiCoP facets (units: meV $\AA^{-2}$ )

\begin{tabular}{lllll}
\hline & $(111)$ & $(100)$ & $(001)$-NiP-t & $(001)$-CoP-t \\
\hline Surface energy & 108.20 & 135.64 & 122.83 & 129.47
\end{tabular}

experiments in which the (111) facet of NiCoP has been synthesized..$^{47-50}$ On the other hand, the surface packing density is usually used to identify the stability. ${ }^{22}$ The surface packing densities of (111), (001)-NiP-t, (001)-CoP-t and (100) facets are $20,17,17$, and 15 atoms per $\mathrm{nm}^{2}$, respectively.

To understand the electronic structures of the four facets, electronic density of states (DOS) data have been calculated, as depicted in Fig. 3. It is found that all facets show metallic behavior with none-zero DOS data at the Fermi level, and the (111) facet has the highest DOS at the Fermi level. It is worth mentioning that the DOS curve for the (100) facet is gentle, indicating that it has small average numbers of electronoccupation states in the valence band because it has a low surface atom packing density and larger interplanar spacing.
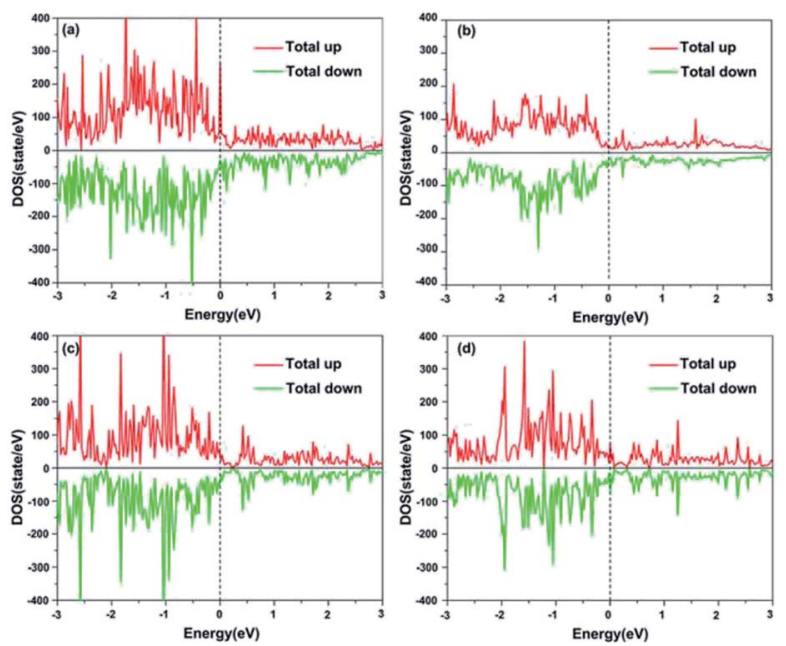

Fig. 3 Spin-polarized total DOS for clean NiCoP facets: (a) (111); (b) (100); (c) (001)-NiP-t; and (d) (001)-CoP-t. The Fermi level is set as zero (dashed line). 


\section{Hydrogen adsorption}

To find the global lowest energy hydrogen adsorption sites, all possible adsorption sites on the four facets were carefully tested. Then the values of $\Delta G_{\mathrm{H}}$ were calculated to describe the HER activity. Finally, $a b$ initio atomistic thermodynamics was used to evaluate the most stable phase for each facet. To systematically understand the HER activity, we will respectively investigate hydrogen adsorption on the four facets.

\section{(111) facet}

The calculated results reveal that there are only three local minimum sites for hydrogen adsorption, named H1, NC2 and P1, as shown in Fig. S1. $\uparrow$ Table S $2 \uparrow$ lists the adsorption energies of each site. It is found that the most favorable adsorption site is $\mathrm{H} 1$. The adsorption energies of all sites follow the trend: $\mathrm{H} 1<$ NC2 $<$ P1. Thus, the initial hydrogen atoms were placed at H1 sites. In the same way, the second lowest energy site was obtained. With an assumption that hydrogen atoms easily spread over the facet at the lowest energy sites for each type of hydrogen coverage, we found the global minimum of hydrogen adsorption for each type of hydrogen coverage on the (111) (a)

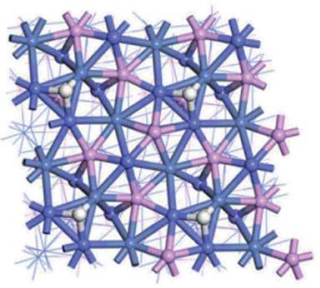

(c)

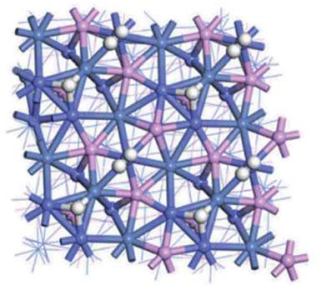

(b)

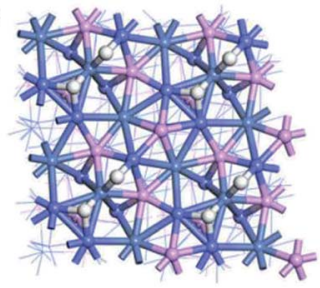

(d)

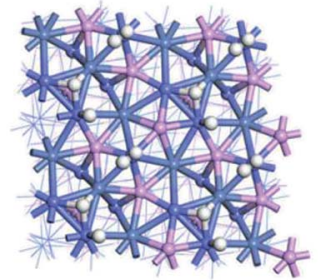

Fig. 4 Optimized structures of the (111) facet for typical hydrogen coverages of (a) $25 \%$, (b) $50 \%$, (c) $75 \%$ and (d) $100 \%$. Note that the colors gray, blue, pink and white stand for $\mathrm{Ni}, \mathrm{Co}, \mathrm{P}$ and $\mathrm{H}$ atoms, respectively. facet, as depicted in Fig. 4 for typical 25\%, 50\%, 75\% and 100\% coverage (here $100 \%$ coverage means $9 \mathrm{H}$ atoms per $\mathrm{nm}^{2}$ ). For less than $25 \%$ hydrogen coverage, hydrogen atoms strongly adsorb on $\mathrm{H} 1$ sites. For $25-50 \%$ hydrogen coverage, they are mainly on NC2 sites. Interestingly, hydrogen atoms on NC2 sites will shift to adjacent $\mathrm{C} 2$ sites when the hydrogen coverage is $56-75 \%$. The distance between proximal hydrogen atoms is $0.875 \AA$, close to the bond length of $0.741 \AA$ found for $\mathrm{H}_{2}$ by Huber et al.,$^{43}$ and they are proposed to react with each other to form $\mathrm{H}_{2}$.

Fig. 5a shows the calculated $\Delta E_{\mathrm{H}}$ and $\Delta G_{\mathrm{H}}$ values for $\mathrm{H}$ adsorption on the (111) facet for different hydrogen coverage. When the hydrogen coverage is in the range of $56-75 \%, \Delta G_{\mathrm{H}}$ is extremely close to zero. Besides, when the hydrogen coverage is $25-50 \%, \Delta G_{\mathrm{H}}$ is close to $-0.2 \mathrm{eV}$, which is a favorable value for the HER. Therefore, it is indicated that the (111) facet can favor the HER over a large hydrogen coverage range.

Furthermore, to calculate the most stable coverage of hydrogen on the NiCoP (111) facet at 1 atm $\mathrm{H}_{2}$ and $298 \mathrm{~K}, a b$ initio atomistic thermodynamics was used to determine $\Delta G^{\text {ad }}(T, P)$ along with the hydrogen chemical potential $\Delta \mu_{\mathrm{H}}$ and pressure, as plotted in Fig. $5 \mathrm{~b}$ and c. Each line expresses a given $\mathrm{H}$ coverage. Clearly, the most stable phase at $1 \mathrm{~atm} \mathrm{H}_{2}$ and $298 \mathrm{~K}$ is $75 \%$ hydrogen coverage, in which the active sites for the HER are $\mathrm{C} 2$ sites.

\section{(100) facet}

Fig. S2 $\uparrow$ shows the hydrogen adsorption sites named N1, NP1, $\mathrm{P} 1$ and so on. The most stable adsorption site is N1, as listed in Table S3. $\dagger$ Fig. S5 $\uparrow$ shows the optimized structures of the (100) facet at typical hydrogen coverages of $25 \%, 50 \%, 75 \%$ and $100 \%$ (here $100 \%$ coverage means $10 \mathrm{H}$ atoms per $\mathrm{nm}^{2}$ ). Because the adsorption sites will change with increasing hydrogen coverage, the NC1 sites can adsorb hydrogen, as mentioned below.

Fig. 6a illustrates $\Delta E_{\mathrm{H}}$ and $\Delta G_{\mathrm{H}}$ values for the (100) facet along with different hydrogen coverage. For $37.5-50 \%$ hydrogen coverage, hydrogen atoms adsorb on NC1 sites with a difference in hydrogen adsorption energy of near $-0.2 \mathrm{eV}$, which is because of interactions between adsorbed hydrogen atoms. Furthermore, values of $\Delta G_{\mathrm{H}}$ close to zero are obtained at NC1 sites for $50 \%$ hydrogen coverage, indicating that the (100) facet
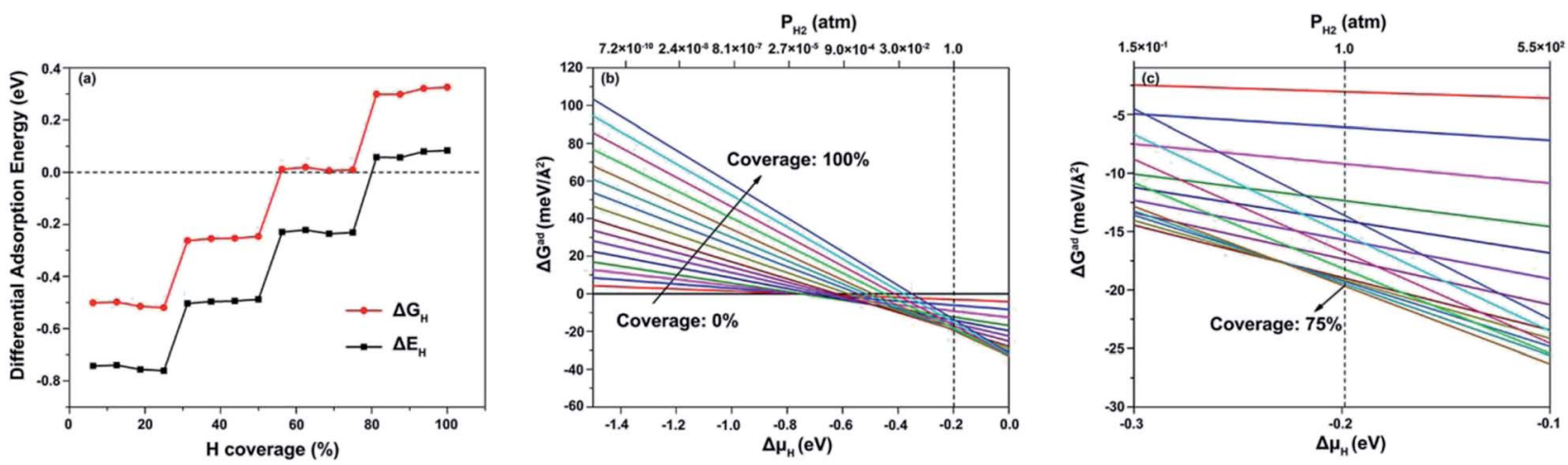

Fig. 5 (a) $\Delta E_{H}$ and $\Delta G_{H}$ values at various hydrogen coverages on the (100) facet; and $\Delta G^{\text {ad }}$ at $298 \mathrm{~K}$ with $\Delta \mu_{\mathrm{H}}$ ranging from (b) -1.5 to 0.0 eV and (c) -0.3 to $-0.1 \mathrm{eV}$. 

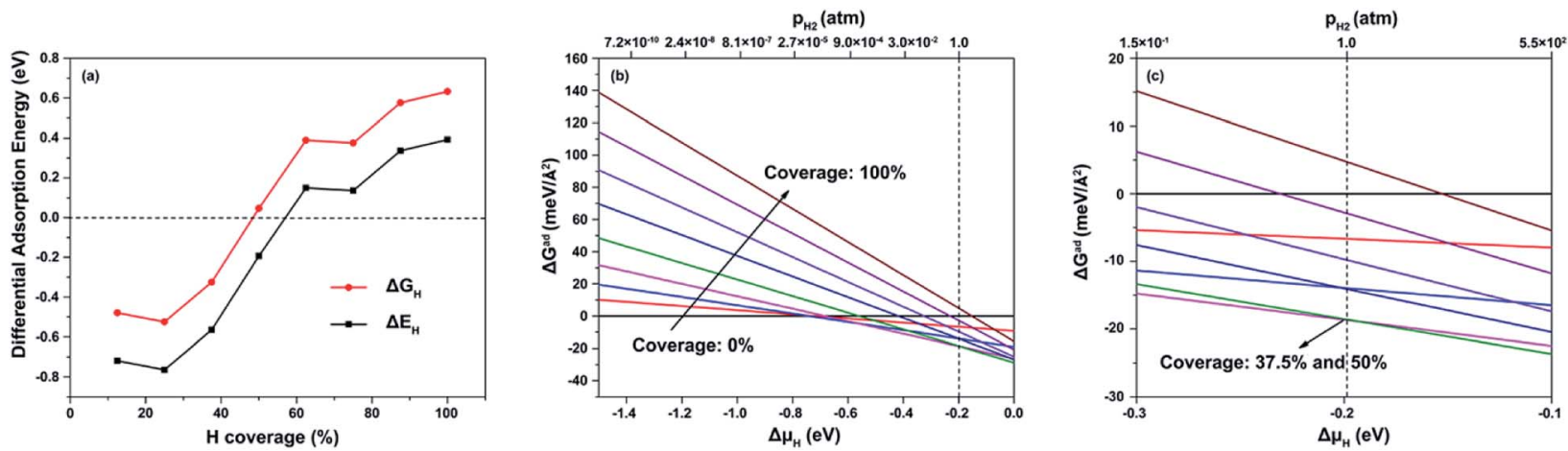

Fig. 6 (a) $\Delta E_{H}$ and $\Delta G_{H}$ values at various hydrogen coverages on the (001)-NiP-t facet; $\Delta G^{\text {ad }}$ at $298 \mathrm{~K}$ with $\Delta \mu_{H}$ ranging from: (b) -1.5 to 0.0 eV and (c) -0.3 to $-0.1 \mathrm{eV}$.

can also be suitable for HER activity, and adsorbed hydrogen atoms have a great influence on the HER. Fig. $6 \mathrm{~b}$ and $\mathrm{c}$ show plots of $\Delta G^{\text {ad }}$ as a function of $\Delta \mu_{\mathrm{H}}$ and pressure at $298 \mathrm{~K}$ for the (100) facet for different hydrogen coverage. It is found that there are two stable hydrogen coverages, $37.5 \%$ and $50 \%$, at $1 \mathrm{H}_{2}$ atm and $298 \mathrm{~K}$, respectively. The main absorption sites are NC1 for such stable hydrogen coverages. Therefore, NC1 sites are the active HER sites for the NiCoP (100) facet with hydrogen coverage of $50 \%$.

\section{(001) facet}

As mentioned above, the (001) facet has two different truncation surfaces called (001)-NiP-t and (001)-CoP-t. Fig. S3 and S4† show the absorption sites for these two facets. The most stable adsorption sites are $\mathrm{H} 2$ and $\mathrm{H} 3$ for (001)-NiP-t (as shown in Table S4 $\dagger$ ) and (001)-CoP-t (as shown in Table S5 $\dagger$ ), respectively. But the adsorption energy of (001)-CoP-t is much larger than that of (001)NiP-t, which indicates that hydrogen adsorption on the (001)CoP-t facet is too strong to desorb $\mathrm{H}_{2}$ at lower hydrogen coverage.

The optimized structures under different hydrogen coverage $\left(100 \%\right.$ coverage represents $7 \mathrm{H}$ atoms per $\mathrm{nm}^{2}$ for (001)-NiP-t and $14 \mathrm{H}$ atoms per $\mathrm{nm}^{2}$ for (001)-CoP-t) are shown in Fig. S6 $\dagger$ for (001)-NiP-t and in Fig. S7† for (001)-CoP-t. It can be found that most active HER sites are $\mathrm{H} 2$ for (001)-NiP-t and $\mathrm{C} 1$ for the (001)-CoP-t facet.

Fig. 7a and 8a plot $\Delta E_{\mathrm{H}}$ and $\Delta G_{\mathrm{H}}$ values at various hydrogen coverages for the (001)-NiP-t and (001)-CoP-t facets. When the hydrogen coverage of the (001)-NiP-t facet is less than $50 \%$, the values of $\Delta G_{\mathrm{H}}$ are close to zero, indicating that the (001)-NiP-t facet has relatively good HER activity with no initial hydrogen coverage, in line with a recent study by Liu et al. ${ }^{51}$ Conversely, the values of $\Delta E_{\mathrm{H}}$ are around zero for the (001)-CoP-t facet when the hydrogen coverage is more than $31 \%$, revealing weak bonding between hydrogen and the facet. Meanwhile, the values of $\Delta G_{\mathrm{H}}$ are close to zero when the hydrogen coverage is more than $31 \%$, illustrating that the hydrogen coverage has an effect on the HER activity and that initial hydrogen coverage is required for favorable HER activity on the (001)-CoP-t facet. Fig. $7(\mathrm{~b}, \mathrm{c})$ and $8(\mathrm{~b}, \mathrm{c})$ depict $\Delta G^{\text {ad }}$ as a function of $\Delta \mu_{\mathrm{H}}$ and pressure at $298 \mathrm{~K}$ for the (001)-NiP-t and (001)-CoP-t facets along with different hydrogen coverage. One can see that the most stable hydrogen coverage at $1 \mathrm{H}_{2}$ atm and $298 \mathrm{~K}$ is $50 \%$ for $(001)$ NiP-t and 25\% for the (001)-CoP-t facet.

\section{HER activity and stability}

Table 3 lists the values of $\Delta G^{\text {ad }}$ and $\Delta G_{\mathrm{H}}$ for the four low Millerindex facets at $1 \mathrm{H}_{2}$ atm and $298 \mathrm{~K}$. We find that durability for the HER follows the trend: (111) > NiCoP(001)-CoP-t > (100) > (001)-NiP-t on the basis of $\Delta G^{\text {ad }}$. Using a $\Delta G_{\mathrm{H}}$ value close to zero as the criterion, the most efficient HER facet is (111). Therefore, from a comprehensive point of view, the (111) facet is the optimal facet for the high-activity HER at $1 \mathrm{H}_{2}$ atm and $298 \mathrm{~K}$.
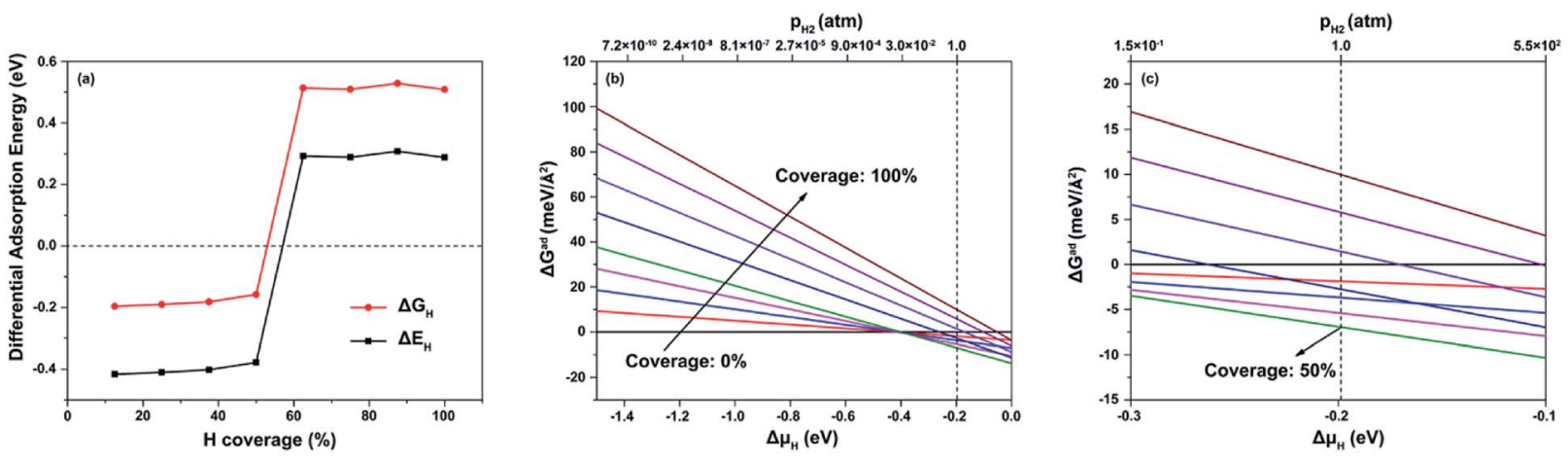

Fig. 7 (a) $\Delta E_{H}$ and $\Delta G_{H}$ values at various hydrogen coverages on the (001)-CoP-t facet; $\Delta G^{\text {ad }}$ at $298 \mathrm{~K}$ with $\Delta \mu_{H}$ ranging from: (b) -1.5 to 0.0 eV and (c) -0.3 to $-0.1 \mathrm{eV}$. 

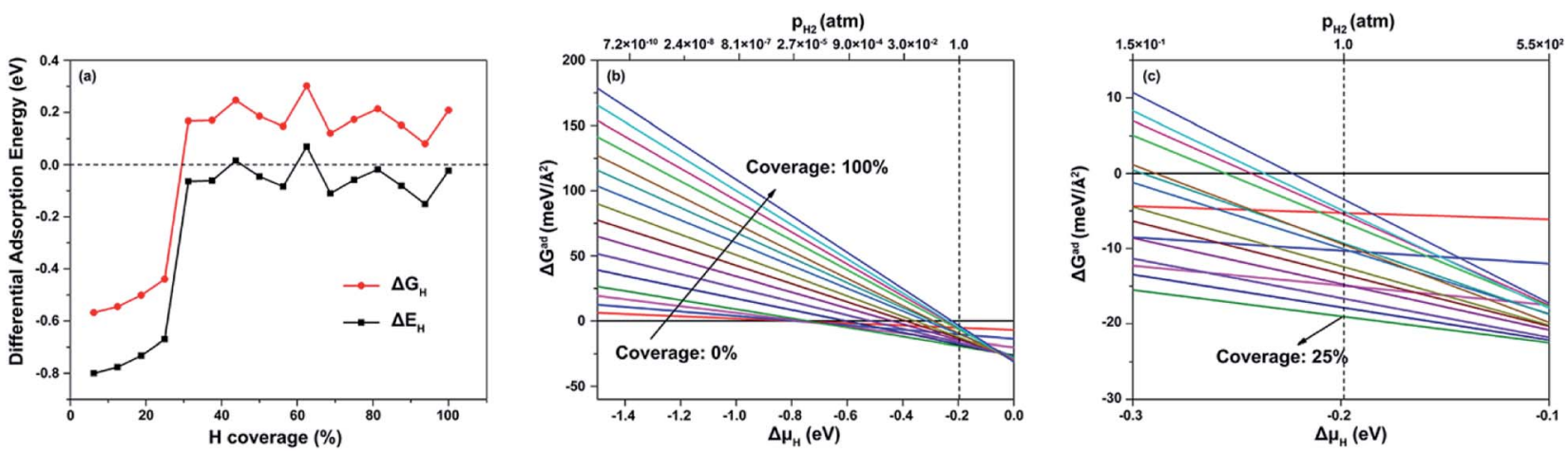

Fig. 8 (a) $\Delta E_{H}$ and $\Delta G_{H}$ values at various hydrogen coverages on the (001)-CoP-t facet; $\Delta G^{\text {ad }}$ at $298 \mathrm{~K}$ with $\Delta \mu_{H}$ ranging from: (b) -1.5 to 0.0 eV and (c) -0.3 to $-0.1 \mathrm{eV}$

Table $3 \Delta G^{\text {ad }}$ and $\Delta G_{H}$ at $1 \mathrm{H}_{2}$ atm and $298 \mathrm{~K}$

\begin{tabular}{lrrlc}
\hline & \multicolumn{1}{c}{$(111)$} & \multicolumn{1}{c}{$(100)$} & $(001)-\mathrm{NiP}-\mathrm{t}$ & $(001)-\mathrm{CoP}-\mathrm{t}$ \\
\hline$\Delta G^{\mathrm{ad}}\left(\mathrm{meV} \mathrm{\AA}^{-2}\right)$ & -19.92 & -18.78 & -7.09 & \multicolumn{1}{c}{19.18} \\
$\Delta G_{\mathrm{H}}(\mathrm{eV})$ & 0.01 & 0.05 & -0.16 & 0.44
\end{tabular}

\section{Conclusions}

In this work, we reported a systematic study of the HER activities of four low Miller-index facets of NiCoP using periodic DFT calculations. The energy electronic structure, Gibbs free energy of hydrogen adsorption and $a b$ initio thermodynamics of hydrogen coverage were investigated to reveal the HER characteristics of NiCoP.

From calculations involving the electronic structures of the surfaces, we found that the stability of the clean facets in a vacuum follows the order: $(111)>(001)-N i P-t>(001)-C o P-t>$ (100), and all facets have metallic behavior with none having zero DOS at the Fermi level. The values of $\Delta G_{\mathrm{H}}$ illustrated that all the calculated facets have good catalytic activities for the HER. When hydrogen coverage is from $56 \%$ to $75 \%$ on the (111) facet, the distance between proximal hydrogen atoms is $0.875 \AA$, which is close to the bond length of $0.741 \AA$ for $\mathrm{H}_{2}$, and they are proposed to react with each other to form $\mathrm{H}_{2}$. From ab initio atomistic thermodynamic analysis, we found that the durability for the HER follows the trend: (111) > (001)-CoP-t > (100) > (001)NiP-t, and the NiCoP (111) facet is the optimal facet for the highactivity $\mathrm{HER}$ at $1 \mathrm{H}_{2}$ atm and $298 \mathrm{~K}$.

\section{Conflicts of interest}

There are no conflicts to declare.

\section{Acknowledgements}

This work was supported by the National Natural Science Foundation of China No. 91641120 and No. 91841301.

\section{References}

1 I. Dincer, Int. J. Energy Res., 2007, 31, 29-55.
2 X. Cheng, Z. Shi, N. Glass, L. Zhang, J. Zhang, D. Song, Z. Liu, H. Wang and J. Shen, J. Power Sources, 2007, 165, 739-756.

3 M. G. Walter, E. L. Warren, J. R. McKone, S. W. Boettcher, Q. Mi, E. A. Santori and N. S. Lewis, Chem. Rev., 2010, 110, 6446-6473.

4 W. Sheng, H. A. Gasteiger and Y. Shao-Horn, J. Electrochem. Soc., 2010, 157, B1529-B1536.

5 Y. Li, H. Wang, L. Xie, Y. Liang, G. Hong and H. Dai, J. Am. Chem. Soc., 2011, 133, 7296-7299.

6 M. A. Abbas and J. H. Bang, Chem. Mater., 2015, 27, 72187235.

7 B. Hinnemann, P. G. Moses, J. Bonde, K. P. N. Jorgensen, J. H. Nielsen, S. Horch, I. Chorkendorff and J. K. Nørskov, J. Am. Chem. Soc., 2005, 127, 5308-5309.

8 T. F. Jaramillo, K. P. Jorgensen, J. Bonde, J. H. Nielsen, S. Horch and I. Chorkendorff, Science, 2007, 317, 100-102.

9 H. Vrubel and X. Hu, Angew. Chem., 2012, 51, 12703-12706.

10 D. Kong, H. Wang, Z. Lu and Y. Cui, J. Am. Chem. Soc., 2014, 136, 4897-4900.

11 W. F. Chen, J. T. Muckerman and E. Fujita, Chem. Commun., 2013, 49, 8896-8909.

12 J. Wang, F. Xu, H. Jin, Y. Chen and Y. Wang, Adv. Mater., 2017, 29, 1605838.

13 W. F. Chen, K. Sasaki, C. Ma, A. I. Frenkel, N. Marinkovic, J. T. Muckerman, Y. Zhu and R. R. Adzic, Angew. Chem., 2012, 51, 6131-6135.

14 S. T. Oyama, T. Gott, H. Zhao and Y. K. Lee, Catal. Today, 2009, 143, 94-107.

15 P. Xiao, W. Chen and X. Wang, Adv. Energy Mater., 2015, 5, 1500985.

16 J. Kibsgaard, C. Tsai, K. Chan, J. D. Benck, J. K. Nørskov, F. Abild-Pedersen and T. F. Jaramillo, Energy Environ. Sci., 2015, 8, 3022-3029.

17 Y. Zhang, H. Lei, D. Duan, E. Villota, C. Liu and R. Ruan, ACS Appl. Mater. Interfaces, 2018, 10, 20429-20439.

18 X. Chen, D. Wang, Z. Wang, P. Zhou, Z. Wu and F. Jiang, Chem. Commun., 2014, 50, 11683-11685.

19 C. Y. Son, I. H. Kwak, Y. R. Lim and J. Park, Chem. Commun., 2016, 52, 2819-2822.

20 P. Jiang, Q. Liu, Y. Liang, J. Tian, A. M. Asiri and X. Sun, Angew. Chem., 2014, 53, 12855-12859. 
21 J. Tian, Q. Liu, A. M. Asiri and X. Sun, J. Am. Chem. Soc., 2014, 136, 7587-7590.

22 G. Hu, Q. Tang and D. E. Jiang, Phys. Chem. Chem. Phys., 2016, 18, 23864-23871.

23 L. A. Stern, L. Feng, F. Song and X. Hu, Energy Environ. Sci., 2015, 8, 2347-2351.

24 E. J. Popczun, J. R. McKone, C. G. Read, A. J. Biacchi, A. M. Wiltrout, N. S. Lewis and R. E. Schaak, J. Am. Chem. Soc., 2013, 135, 9267-9270.

25 H. Liang, A. N. Gandi, D. H. Anjum, X. Wang, U. Schwingenschlogl and H. N. Alshareef, Nano Lett., 2016, 16, 7718-7725.

26 Y. Li, J. Liu, C. Chen, X. Zhang and J. Chen, ACS Appl. Mater. Interfaces, 2017, 9, 5982-5991.

27 Y. Feng, X. Y. Yu and U. Paik, Chem. Commun., 2016, 52, 1633-1636.

28 Y. Bai, H. Zhang, L. Liu, H. Xu and Y. Wang, Chem.-Eur. J., 2016, 22, 1021-1029.

29 C. Wang, J. Jiang, T. Ding, G. Chen, W. Xu and Q. Yang, Adv. Mater. Interfaces, 2016, 3, 1500454.

30 M. Kong, Z. Wang, W. Wang, M. Ma, D. Liu, S. Hao, R. Kong, G. Du, A. M. Asiri, Y. Yao and X. Sun, Chem.-Eur. J., 2017, 23, 4435-4441.

31 J. Li, G. Wei, Y. Zhu, Y. Xi, X. Pan, Y. Ji, I. V. Zatovsky and W. Han, J. Mater. Chem. A, 2017, 5, 14828-14837.

32 J. Li, M. Yan, X. Zhou, Z. Q. Huang, Z. Xia, C.-R. Chang, Y. Ma and Y. Qu, Adv. Funct. Mater., 2016, 26, 6785-6796.

33 T. Volmer and M. Z. Erdey-Gruz, Phys. Chem. A, 1930, 150, 203-213.

34 J. Heyrovsky, Recl. Trav. Chim. Pays-Bas, 1927, 46, 582-585.

35 J. Z. Tafel, Phys. Chem. A, 1905, 50, 641-712.

36 R. Parsons, Trans. Faraday Soc., 1958, 54, 1053-1063.
37 J. K. Nørskov, T. Bligaard, A. Logadottir, J. R. Kitchin, J. G. Chen, S. Pandelov and U. Stimming, J. Electrochem. Soc., 2005, 152, J23-J26.

38 J. Greeley, T. F. Jaramillo, J. Bonde, I. B. Chorkendorff and J. K. Norskov, Nat. Mater., 2005, 5, 909-913.

39 G. Kresse and J. Hafner, Phys. Rev. B: Condens. Matter Mater. Phys., 1993, 47, 558-561.

40 G. Kresse and D. Joubert, Phys. Rev. B: Condens. Matter Mater. Phys., 1999, 59, 1758-1775.

41 P. E. Blöchl, Phys. Rev. B: Condens. Matter Mater. Phys., 1994, 50, 17953-17979.

42 J. P. Perdew and K. Burke, Phys. Rev. Lett., 1996, 77, 38653868.

43 K. P. Huber and G. Herzberg, Molecular structure and molecular spectra IV: constants of diatomic molecules, Van Nostrand-Reinhold, New York, 1979.

44 D. R. Stull and H. Prophet, Natl. Stand. Ref. Data Ser., 1971, 35,5 .

45 J. P. Sénateur, A. Rouault, P. L’Héritier, A. F. KrumbugelNylund, R. Fruchart, D. Fruchart, P. Convert and E. Roudaut, Mater. Res. Bull., 1973, 8, 229-238.

46 Z. Liang, X. Zhong, T. Li, M. Chen and G. Feng, ChemElectroChem, 2019, 6, 260-267.

47 Z. Cai, A. Wu, H. Yan, Y. Xiao, C. Chen, C. Tian, L. Wang, R. Wang and H. Fu, Nanoscale, 2018, 10, 7619-7629.

48 L. Su, H. Li, Y. Xiao, G. Han and M. Zhu, J. Alloys Compd., 2019, 771, 117-123.

49 T. Liu, X. Yan, P. Xi, J. Chen, D. Qin, D. Shan, S. Devaramani and X. Lu, Int. J. Hydrogen Energy, 2017, 42, 14124-14132.

50 C. Wang, J. Jiang, T. Ding, G. Chen, W. Xu and Q. Yang, Adv. Mater. Interfaces, 2016, 3, 1500454.

51 J. Liu, Z. Wang, J. David, J. Llorca, J. Li, X. Yu, A. Shavel, J. Arbiol, M. Meyns and A. Cabot, J. Mater. Chem. A, 2018, 6, 11453-11462. 